\title{
Catalytic Intermolecular Amination of C-H Bonds: Method Development and Mechanistic Insights
}

\section{Supplementary Material}

(12 pages)

Kristin Williams Fiori and J. Du Bois*

Department of Chemistry

Stanford University

Stanford, CA 94305-5080 
General. All reagents were obtained commercially unless otherwise noted. Reactions were performed using ovendried glassware under an atmosphere of nitrogen. Organic solutions were concentrated under reduced pressure $(\sim 15$ Torr) by rotary evaporation. Freshly distilled solvents were employed unless otherwise noted. Dichloromethane, benzene, and toluene were dried by passage through activated alumina columns under 12 psi of $\mathrm{N}_{2}$. The preparation of $\mathrm{Rh}_{2}(\mathrm{esp})_{2}$ followed a previously published protocol. ${ }^{1}$

Chromatographic purification of products was accomplished using forced-flow chromatography on EM Science Geduran silica gel $60(35-75 \mu \mathrm{m})$. Thin-layer chromatography (TLC) was performed on EM Science silica gel $60 \mathrm{~F}_{254}$ plates $(250 \mu \mathrm{m})$. Visualization of the developed chromatogram was accomplished by fluorescence quenching and by staining with ethanolic anisaldehyde, aqueous potassium permanganate, or aqueous ceric ammonium molybdate (CAM) solution.

Nuclear magnetic resonance (NMR) spectra were acquired on a Varian Mercury spectrometer operating at 400 and $100 \mathrm{MHz}$ for ${ }^{1} \mathrm{H}$ and ${ }^{13} \mathrm{C}$, respectively, or on a Varian Inova spectrometer operating at 500 and $125 \mathrm{MHz}$ for ${ }^{1} \mathrm{H}$ and ${ }^{13} \mathrm{C}$, respectively, and are referenced internally according to residual solvent signals. Data for ${ }^{1} \mathrm{H}$ NMR are recorded as follows: chemical shift $(\delta$, ppm), multiplicity (s, singlet; br s, broad singlet; d, doublet; br d, broad doublet; t, triplet; q, quartet; quint, quintet; sept, septet; m, multiplet), integration, and coupling constant (Hz). Data for ${ }^{13} \mathrm{C}$ NMR are reported in terms of chemical shift $(\delta, \mathrm{ppm})$. Infrared spectra were recorded on a Thermo Nicolet IR300 spectrometer and are reported in terms of frequency of absorption. Sample preparation was done either as a thin film on a $\mathrm{NaCl}$ salt plate or as a $\mathrm{KBr}$ pellet. UV/Visible spectra were collected on a Varian Cary 6000i UV-visNIR spectrophotometer. High-resolution mass spectra were obtained from the Vincent Coates Foundation Mass Spectrometry Laboratory at Stanford University.

$\operatorname{PhI}\left(\mathrm{O}_{2} \mathbf{C}^{t} \mathbf{B u}\right)_{2}:{ }^{2}$ A $500 \mathrm{~mL}$ round bottom flask was charged with $\mathrm{PhI}(\mathrm{OAc})_{2}(15.0 \mathrm{~g}, 46.5 \mathrm{mmol}), 180 \mathrm{~mL}$ of chlorobenzene, and trimethylacetic acid $(16.5 \mathrm{~g}, 0.16 \mathrm{~mol}, 3.0$ equiv). The flask was fitted with a short-path distillation head and a $250 \mathrm{~mL}$ receiving flask. The reaction was stirred at $50{ }^{\circ} \mathrm{C}$ under reduced pressure (30 torr) and the chlorobenzene was slowly distilled (ca. 8-10 h) until $\sim 20-30 \mathrm{~mL}$ of solvent remained. After cooling to 23 ${ }^{\circ} \mathrm{C}, 150 \mathrm{~mL}$ of hexanes was added to precipitate a white solid. The desired product was collected upon filtration and dried in vacuo $(17.9 \mathrm{~g}, 95 \%)$. ${ }^{1} \mathrm{H}$ NMR $\left(\mathrm{CDCl}_{3}, 400 \mathrm{MHz}\right) \delta$ 8.02-7.99 (m, 2H), 7.57-7.52 (m, 1H), 7.50-7.44 (m, $2 \mathrm{H}), 1.11(\mathrm{~s}, 18 \mathrm{H}) \mathrm{ppm} ;{ }^{13} \mathrm{C} \mathrm{NMR}\left(\mathrm{CDCl}_{3}, 100 \mathrm{MHz}\right) \delta 183.5,134.2,131.2,130.6,122.0,38.9,27.7 \mathrm{ppm}$; IR (thin film) $v 2974,2931,2870,1644,1477,1443,1393,1364,1291,1175,994,890 \mathrm{~cm}^{-1}$.

$\mathbf{H}_{2} \mathbf{N S O}_{3} \mathbf{C H}_{2} \mathbf{C C l}_{3}:{ }^{3}$ To a $100 \mathrm{~mL}$ three-neck flask equipped with a $25 \mathrm{~mL}$ addition funnel, a $\mathrm{CaSO}_{4}$-filled drying tube, and a rubber septum were added $10 \mathrm{~mL}$ of $\mathrm{CH}_{3} \mathrm{CN}$ and $\mathrm{ClSO}_{2} \mathrm{NCO}(2.7 \mathrm{~mL}, 31.3 \mathrm{mmol}, 1.5$ equiv). After cooling the solution to $0{ }^{\circ} \mathrm{C}, \mathrm{HCO}_{2} \mathrm{H}\left(1.2 \mathrm{~mL}, 31.3 \mathrm{mmol}, 1.5\right.$ equiv) in $6 \mathrm{~mL}$ of $\mathrm{CH}_{3} \mathrm{CN}$ was added dropwise from the addition funnel over a 10 min period. Following this time, the mixture was warmed to $23{ }^{\circ} \mathrm{C}$ and stirred for $8 \mathrm{~h}$. The reaction vessel was then returned to the ice bath before a solution of 2,2,2-trichloroethanol ( $2.0 \mathrm{~mL}, 20.8 \mathrm{mmol})$ in $12 \mathrm{~mL}$ of DMA was added via cannula. Transfer of the alcohol was made quantitative with an additional $4 \mathrm{~mL}$ of DMA. The contents were warmed to $23{ }^{\circ} \mathrm{C}$ and stirred vigorously for $1.5 \mathrm{~h}$. The reaction mixture was quenched by the addition of $50 \mathrm{~mL}$ of $\mathrm{H}_{2} \mathrm{O}$ and poured into a separatory funnel containing $100 \mathrm{~mL}$ of $\mathrm{Et}_{2} \mathrm{O}$. The organic phase was collected and the aqueous layer was extracted with $2 \times 100 \mathrm{~mL}$ of $\mathrm{Et}_{2} \mathrm{O}$. The combined ethereal extracts were washed with $5 \times 50 \mathrm{~mL}$ of $\mathrm{H}_{2} \mathrm{O}$, dried over $\mathrm{MgSO}_{4}$, and concentrated under reduced pressure. Purification of the oily residue by chromatography on silica gel using 2:1 hexanes/EtOAc as eluent afforded the desired product as a white solid (4.35 g, 91\%). TLC $\mathrm{R}_{f}=0.35\left(15: 1 \mathrm{CH}_{2} \mathrm{Cl}_{2} / \mathrm{EtOAc}\right) ; \mathrm{mp}=52-54{ }^{\circ} \mathrm{C} ;{ }^{1} \mathrm{H}$ NMR $\left(\mathrm{CDCl}_{3}, 400 \mathrm{MHz}\right) \delta$ 5.40 (br s, 2H), 4.69 (s, 2H) ppm; ${ }^{13} \mathrm{C} \mathrm{NMR}\left(\mathrm{CDCl}_{3}, 100 \mathrm{MHz}\right) \delta 93.0,78.6 \mathrm{ppm}$; IR (thin film) v 3404, 3296, 3095, 1551, 1371, 1267, 1189, 1089, 1016, 932, 860, $770 \mathrm{~cm}^{-1}$; HRMS (EI) calcd for $\mathrm{C}_{2} \mathrm{H}_{4} \mathrm{Cl}_{3} \mathrm{NO}_{3} \mathrm{~S} 226.8977$ found $227.9069\left(\mathrm{MH}^{+}\right)$. 
Preparation of $\mathbf{H}_{2}{ }^{15} \mathrm{NSO}_{3} \mathbf{C H}_{2} \mathbf{C C l}_{3}$ : A $50 \mathrm{~mL}$ 2-neck flask fitted with a gas inlet (glass pipette) and a rubber septum was charged with $7 \mathrm{~mL}$ of THF and $\mathrm{Cl}_{3} \mathrm{CCH}_{2} \mathrm{OSO}_{2} \mathrm{Cl}(0.60 \mathrm{~g}, 2.4 \mathrm{mmol}) .{ }^{4}$ The solution was cooled to $0{ }^{\circ} \mathrm{C}$ and gaseous ${ }^{15} \mathrm{NH}_{3}$ was bubbled into the mixture for $15 \mathrm{~s}$. A white precipitate formed almost immediately upon addition of ${ }^{15} \mathrm{NH}_{3}$. The gas inlet was quickly removed and the reaction flask was sealed with a glass stopper. The white suspension stirred vigorously while the contents slowly warmed from $0^{\circ}$ to $23{ }^{\circ} \mathrm{C}$ over the course of $1 \mathrm{~h}$. The reaction mixture was then quenched with $10 \mathrm{~mL}$ of $\mathrm{H}_{2} \mathrm{O}$, transferred to a separatory funnel, and extracted with $2 \mathrm{x}$ $25 \mathrm{~mL}$ of $\mathrm{Et}_{2} \mathrm{O}$. The organic extracts were combined and washed with $1 \times 10 \mathrm{~mL}$ of saturated aqueous $\mathrm{NaCl}$, dried over $\mathrm{MgSO}_{4}$, and concentrated under reduced pressure. Purification of the oily residue by chromatography on silica gel (3:1 hexanes/EtOAc) afforded the desired sulfamate as a white solid (420 mg, 76\%). TLC $\mathrm{R}_{f}=0.35(15: 1$ $\left.\mathrm{CH}_{2} \mathrm{Cl}_{2} / \mathrm{EtOAc}\right) ;{ }^{1} \mathrm{H}$ NMR $\left(\mathrm{CDCl}_{3}, 400 \mathrm{MHz}\right) \delta 5.00\left(\mathrm{~d}, 2 \mathrm{H}, J_{\mathrm{NH}}=83.5 \mathrm{~Hz}\right), 4.71(\mathrm{~s}, 2 \mathrm{H}) \mathrm{ppm} ;{ }^{13} \mathrm{C} \mathrm{NMR}\left(\mathrm{CDCl}_{3}\right.$, $100 \mathrm{MHz}$ ) $\delta$ 94.4, $78.7 \mathrm{ppm}$; IR (thin film) v 3287, 2957, 1619, 1547, 1448, 1371, 1267, 1188, 1089, 1047, 1016, $921,858,769 \mathrm{~cm}^{-1}$; HRMS $\left(\mathrm{ES}^{+}\right)$calcd for $\mathrm{C}_{2} \mathrm{H}_{4} \mathrm{Cl}_{3}{ }^{15} \mathrm{NO}_{3} \mathrm{~S} 227.8947$ found $250.8850\left(\mathrm{MNa}^{+}\right)$.

\section{Experimental procedure and characterization data intermolecular $\mathrm{C}-\mathrm{H}$ amination reactions}

A flask containing $\mathrm{H}_{2} \mathrm{NSO}_{3} \mathrm{CH}_{2} \mathrm{CCl}_{3}(137 \mathrm{mg}, 0.60 \mathrm{mmol})$ in $0.6 \mathrm{~mL}$ of $\mathrm{C}_{6} \mathrm{H}_{6}$ was charged with $\mathrm{Rh}_{2}(\mathrm{esp})_{2}(9 \mathrm{mg}, 12$ $\mu \mathrm{mol}, 0.02$ equiv) and substrate $(0.60 \mathrm{mmol})$. To this bright green mixture was added $1.4 \mathrm{~mL}$ of a $0.83 \mathrm{M} \mathrm{C}_{6} \mathrm{H}_{6}$ solution of $\mathrm{PhI}\left(\mathrm{O}_{2} \mathrm{C}^{\mathrm{t} B u}\right)_{2}\left(1.2 \mathrm{mmol}, 2.0\right.$ equiv) via syringe pump over $3 \mathrm{~h}$. During the addition of $\mathrm{PhI}\left(\mathrm{O}_{2} \mathrm{C} B \mathrm{Bu}\right)_{2}$, a change in the reaction color to brown or red was generally observed. Following the transfer of oxidant, the solution was stirred at $23{ }^{\circ} \mathrm{C}$ for $1-2 \mathrm{~h}$. Dichloromethane $(5 \mathrm{~mL})$ and $2 \mathrm{~mL}$ of a saturated aqueous solution of thiourea were then added and the orange biphasic mixture was stirred vigorously for $30 \mathrm{~min}^{5}$ The contents were transferred to a separatory funnel and the organic phase was collected. The aqueous layer was extracted with $2 \times 10 \mathrm{~mL}$ of $\mathrm{CH}_{2} \mathrm{Cl}_{2}$. The combined organic extracts were washed with $2 \times 10 \mathrm{~mL}$ of a $0.1 \mathrm{M} \mathrm{pH} 7 \mathrm{Na}_{2} \mathrm{HPO}_{4} / \mathrm{NaH}_{2} \mathrm{PO}_{4}$ buffer, dried over $\mathrm{MgSO}_{4}$, and concentrated under reduced pressure. Purification of the isolated material by chromatography on silica gel (conditions given below) afforded the desired product.

\footnotetext{
$\mathrm{Ph} \stackrel{\mathrm{NHSO}_{3} \mathrm{CH}_{2} \mathrm{CCl}_{3}}{\stackrel{\mathrm{Me}}{\mathrm{P}}}$
}

Purified by chromatography on silica gel (9:1 hexanes/EtOAc); pale yellow oil (72\%): TLC $\mathrm{R}_{f}=0.38$ (4:1 hexanes/EtOAc); ${ }^{1} \mathrm{H} \mathrm{NMR}\left(\mathrm{CDCl}_{3}, 400 \mathrm{MHz}\right) \delta 7.41-7.30(\mathrm{~m}, 5 \mathrm{H}), 4.89$ (br d, $1 \mathrm{H}, J=6.8 \mathrm{~Hz}$ ), 4.74 (quint, $1 \mathrm{H}, J=$ $6.8 \mathrm{~Hz}), 4.44(\mathrm{~d}, 1 \mathrm{H}, J=10.8 \mathrm{~Hz}), 4.43(\mathrm{~d}, 1 \mathrm{H}, J=10.8 \mathrm{~Hz}), 1.63(\mathrm{~d}, 3 \mathrm{H}, J=6.8 \mathrm{~Hz}) \mathrm{ppm} ;{ }^{13} \mathrm{C} \mathrm{NMR}\left(\mathrm{CDCl}_{3}, 100\right.$ MHz) $\delta 141.3,129.0,128.3,126.2,93.2,78.1,54.9,22.8$ ppm; IR (thin film) v 3304, 3066, 3033, 2981, 2950, 1496, $1433,1365,1273,1182,1121,1086,1047,1015,976,854 \mathrm{~cm}^{-1}$; HRMS (ES ${ }^{+}$calcd for $\mathrm{C}_{10} \mathrm{H}_{12} \mathrm{Cl}_{3} \mathrm{NO}_{3} \mathrm{~S} 330.9603$ found $353.9503\left(\mathrm{MNa}^{+}\right)$.

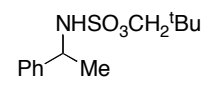

Purified by chromatography on silica gel (9:1 hexanes/EtOAc); pale yellow oil (47\%): TLC $\mathrm{R}_{f}=0.41$ (4:1 hexanes/EtOAc); ${ }^{1} \mathrm{H} \mathrm{NMR}\left(\mathrm{CDCl}_{3}, 400 \mathrm{MHz}\right) \delta$ 7.38-7.33 (m, 3H), 7.33-7.28 (m, 2H), 4.64 (quint, $1 \mathrm{H}, J=6.7 \mathrm{~Hz}$ ), $4.58(\mathrm{br} \mathrm{d}, 1 \mathrm{H}, J=6.7 \mathrm{~Hz}), 3.68(\mathrm{~d}, 1 \mathrm{H}, J=8.8 \mathrm{~Hz}), 3.57(\mathrm{~d}, 1 \mathrm{H}, J=8.8 \mathrm{~Hz}), 1.58(\mathrm{~d}, 3 \mathrm{H}, J=6.7 \mathrm{~Hz}) 0.83(\mathrm{~s}, 9 \mathrm{H})$ ppm; ${ }^{13} \mathrm{C}$ NMR $\left(\mathrm{CDCl}_{3}, 100 \mathrm{MHz}\right) \delta 142.3,128.7,127.8,126.1,79.4,54.3,31.3,25.9,23.3 \mathrm{ppm}$; IR (thin film) $v$ $3292,2961,1460,1433,1357,1174,1122,1086,963,880,826 \mathrm{~cm}^{-1}$; HRMS (ES ${ }^{+}$) calcd for $\mathrm{C}_{13} \mathrm{H}_{21} \mathrm{NO}_{3} \mathrm{~S} 271.1242$ found $294.1138\left(\mathrm{MNa}^{+}\right)$. 


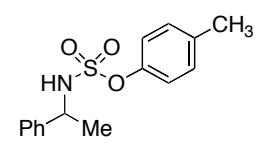

Purified by chromatography on silica gel (9:1 hexanes/EtOAc); pale yellow oil (20\%): TLC $\mathrm{R}_{f}=0.35$ (4:1 hexanes/EtOAc); ${ }^{1} \mathrm{H}$ NMR ( $\left.\mathrm{CDCl}_{3}, 400 \mathrm{MHz}\right) \delta$ 7.40-7.30 (m, 5H), 7.11-7.07 (m, 2H), 6.98-6.94 (m, 2H), 5.03 (br d, $1 \mathrm{H}, J=6.9 \mathrm{~Hz}$ ), 4.74 (quint, $1 \mathrm{H}, J=6.9 \mathrm{~Hz}), 2.32(\mathrm{~s}, 3 \mathrm{H}), 1.59$ (d, $3 \mathrm{H}, J=6.9 \mathrm{~Hz}) \mathrm{ppm} ;{ }^{13} \mathrm{C} \mathrm{NMR}\left(\mathrm{CDCl}_{3}, 100\right.$ MHz) $\delta 147.8,141.7,136.6,130.1,128.8,128.1,126.2,121.5,55.0,23.1,20.8$ ppm; IR (thin film) v 3299, 3033, 2980, 2927, 1504, 1456, 1432, 1364, 1197, 1176, 1152, 1120, 1105, 1084, 1020, 1001, 976, 869, 831, $795 \mathrm{~cm}^{-1}$; HRMS (ES ${ }^{+}$) calcd for $\mathrm{C}_{15} \mathrm{H}_{17} \mathrm{NO}_{3} \mathrm{~S} 291.0929$ found $314.0839\left(\mathrm{MNa}^{+}\right)$.

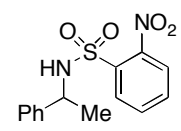

Purified by chromatography on silica gel (gradient elution: 9:1 $\rightarrow 4: 1$ hexanes/EtOAc); white solid (29\%): $\mathrm{TLC}_{f}=$ 0.35 (2:1 hexanes/EtOAc); $\mathrm{mp}=84-88{ }^{\circ} \mathrm{C} ;{ }^{1} \mathrm{H} \mathrm{NMR}\left(\mathrm{CDCl}_{3}, 400 \mathrm{MHz}\right) \delta 7.71(\mathrm{dd}, 1 \mathrm{H}, J=7.9,1.2 \mathrm{~Hz}), 7.66$ (dd, $1 \mathrm{H}, J=7.8,1.4 \mathrm{~Hz}), 7.53(\mathrm{td}, 1 \mathrm{H}, J=7.9,1.4 \mathrm{~Hz}), 7.40(\mathrm{td}, 1 \mathrm{H}, J=7.8,1.2 \mathrm{~Hz}), 7.12-7.05(\mathrm{~m}, 5 \mathrm{H}), 5.78(\mathrm{br} \mathrm{d}, 1 \mathrm{H}$, $J=8.4 \mathrm{~Hz}), 4.69(\mathrm{dq}, 1 \mathrm{H}, J=8.4,7.0 \mathrm{~Hz}), 1.52(\mathrm{~d}, 3 \mathrm{H}, J=7.0 \mathrm{~Hz}) \mathrm{ppm} ;{ }^{13} \mathrm{C}$ NMR $\left(\mathrm{CDCl}_{3}, 100 \mathrm{MHz}\right) \delta 147.2$, 141.1, 134.3, 132.9, 132.4, 130.7, 128.4, 127.6, 126.0, 124.8, 54.9, 23.6 ppm; IR (thin film) v 3347, 3096, 3030, 2979, 2927, 1594, 1540, 1496, 1456, 1415, 1361, 1339, 1302, 1208, 1170, 1119, 1084, 1061, 1017, 1000, 961, 854 $\mathrm{cm}^{-1}$; HRMS (ES) calcd for $\mathrm{C}_{14} \mathrm{H}_{14} \mathrm{~N}_{2} \mathrm{O}_{4} \mathrm{~S} 306.0674$ found $329.0574\left(\mathrm{MNa}^{+}\right)$.

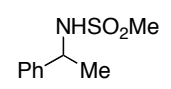

Purified by chromatography on silica gel (3:1 hexanes/EtOAc); pale yellow oil (20\%): TLC $\mathrm{R}_{f}=0.26$ (2:1 hexanes/EtOAc); ${ }^{1} \mathrm{H}$ NMR $\left(\mathrm{CDCl}_{3}, 400 \mathrm{MHz}\right) \delta$ 7.40-7.28 (m, 5H), 4.95 (br s, $\left.1 \mathrm{H}\right), 4.64$ (quint, $1 \mathrm{H}, J=6.9 \mathrm{~Hz}$ ), $2.61(\mathrm{~s}, 3 \mathrm{H}), 1.54(\mathrm{~d}, 3 \mathrm{H}, J=6.9 \mathrm{~Hz}) \mathrm{ppm} ;{ }^{13} \mathrm{C} \mathrm{NMR}\left(\mathrm{CDCl}_{3}, 100 \mathrm{MHz}\right) \delta 142.4,128.9,128.0,126.2,53.7,41.7$, $24.0 \mathrm{ppm}$; IR (thin film) v 3278, 2977, 2932, 1495, 1454, 1316, 1152, 1120, 1086, 1021, 981, $862 \mathrm{~cm}^{-1}$; HRMS $\left(\mathrm{ES}^{+}\right)$calcd for $\mathrm{C}_{9} \mathrm{H}_{13} \mathrm{NO}_{2} \mathrm{~S} 199.0667$ found $222.0556\left(\mathrm{MNa}^{+}\right)$.

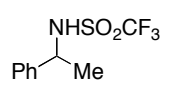

Purified by chromatography on silica gel (9:1 hexanes/EtOAc); pale yellow oil (35\%): TLC $\mathrm{R}_{f}=0.69$ (2:1 hexanes/EtOAc); ${ }^{1} \mathrm{H}$ NMR ( $\left.\mathrm{CDCl}_{3}, 400 \mathrm{MHz}\right) \delta$ 7.42-7.36 (m, 2H), 7.36-7.30 (m, 3H), 5.24 (br s, 1H), 4.80 (quint, $1 \mathrm{H}, J=7.0 \mathrm{~Hz}), 1.64(\mathrm{~d}, 3 \mathrm{H}, J=6.9 \mathrm{~Hz}) \mathrm{ppm} ;{ }^{13} \mathrm{C} \mathrm{NMR}\left(\mathrm{CDCl}_{3}, 100 \mathrm{MHz}\right) \delta 140.9,129.0,128.4,125.7,119.5(\mathrm{q}$, $J_{\mathrm{CF}}=320.4 \mathrm{~Hz}$ ), 55.3, $23.4 \mathrm{ppm}$; IR (thin film) $v$ 3307, 1433, 1372, 1231, 1195, 1147, 1080, 1023, 980, 868 $\mathrm{cm}^{-1}$.

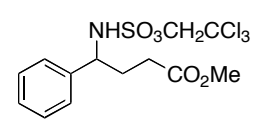

Purified by chromatography on silica gel (gradient elution: 9:1 $\rightarrow 4: 1$ hexanes/EtOAc); clear oil (70\%): $\mathrm{TLC} \mathrm{R}_{f}=$ $0.48\left(2: 1\right.$ hexanes/EtOAc); ${ }^{1} \mathrm{H}$ NMR $\left(\mathrm{CDCl}_{3}, 400 \mathrm{MHz}\right) \delta 7.40-7.35(\mathrm{~m}, 2 \mathrm{H}), 7.33-7.29(\mathrm{~m}, 3 \mathrm{H}), 5.87(\mathrm{br} \mathrm{d}, 1 \mathrm{H}, J=$ $7.7 \mathrm{~Hz}), 4.58(\mathrm{dt}, 1 \mathrm{H}, J=7.7,6.3 \mathrm{~Hz}), 4.34(\mathrm{~d}, 1 \mathrm{H}, J=10.8 \mathrm{~Hz}), 4.29(\mathrm{~d}, 1 \mathrm{H}, J=10.8 \mathrm{~Hz}), 3.69(\mathrm{~s}, 3 \mathrm{H}), 2.51-2.38$ $(\mathrm{m}, 2 \mathrm{H}), 2.30-2.20(\mathrm{~m}, 1 \mathrm{H}), 2.12(\mathrm{dq}, 1 \mathrm{H}, J=14.3,6.8 \mathrm{~Hz}) \mathrm{ppm} ;{ }^{13} \mathrm{C} \mathrm{NMR}\left(\mathrm{CDCl}_{3}, 100 \mathrm{MHz}\right) \delta 174.2,140.0$, 129.0, 128.4, 126.5, 93.2, 77.9, 59.0, 52.1, 31.5, 30.6 ppm; IR (thin film) v 3286, 2954, 1717, 1440, 1369, 1260, 1182, 1089, 1048, 1019, 990, 910, $855 \mathrm{~cm}^{-1}$; HRMS $\left(\mathrm{ES}^{+}\right)$calcd for $\mathrm{C}_{13} \mathrm{H}_{10} \mathrm{Cl}_{3} \mathrm{NO}_{5} \mathrm{~S} 402.9815$ found 425.9699 $\left(\mathrm{MNa}^{+}\right)$. 


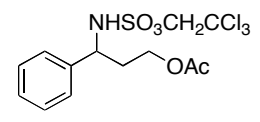

Purified by chromatography on silica gel (gradient elution: 9:1 $\rightarrow 4: 1$ hexanes/EtOAc); white solid (74\%): $\mathrm{TLC} \mathrm{R}_{f}=$ 0.42 (2:1 hexanes/EtOAc); $\mathrm{mp}=99-103{ }^{\circ} \mathrm{C} ;{ }^{1} \mathrm{H}$ NMR $\left(\mathrm{CDCl}_{3}, 400 \mathrm{MHz}\right) \delta$ 7.42-7.36 (m, 2H), 7.35-7.29 (m, 3H), 5.59 (br d, $1 \mathrm{H}, J=7.6 \mathrm{~Hz}), 4.68(\mathrm{q}, 1 \mathrm{H}, J=7.6 \mathrm{~Hz}), 4.34$ (d, $1 \mathrm{H}, J=10.8 \mathrm{~Hz}), 4.29$ (d, 1H, $J=10.8 \mathrm{~Hz}), 4.15$ (ddd, $1 \mathrm{H}, J=11.5,6.7,5.4 \mathrm{~Hz}), 4.03(\mathrm{ddd}, 1 \mathrm{H}, J=11.5,7.0,5.3 \mathrm{~Hz}), 2.29(\mathrm{dddd}, 1 \mathrm{H}, J=14.3,7.0,7.0,5.4 \mathrm{~Hz}), 2.16$ (dddd, $1 \mathrm{H}, J=14.3,14.3,6.7,5.3 \mathrm{~Hz}), 2.04$ (s, 3H) ppm; ${ }^{13} \mathrm{C} \mathrm{NMR}\left(\mathrm{CDCl}_{3}, 100 \mathrm{MHz}\right) \delta 171.1,139.5,129.1,128.5$, 126.6, 93.2, 78.0, 61.0, 56.7, 35.5, 20.9 ppm; IR (thin film) v 3281, 1716, 1459, 1369, 1264, 1183, 1088, 1046, $1019,932,856 \mathrm{~cm}^{-1}$; HRMS $\left(\mathrm{ES}^{+}\right)$calcd for $\mathrm{C}_{13} \mathrm{H}_{16} \mathrm{Cl}_{3} \mathrm{NO}_{5} \mathrm{~S} 402.9815$ found $425.9716\left(\mathrm{MNa}^{+}\right)$.

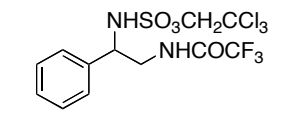

Purified by chromatography on silica gel (gradient elution: 50:1 $\rightarrow 20: 1 \mathrm{CH}_{2} \mathrm{Cl}_{2} /$ EtOAc); off-white solid (65\%): TLC $\mathrm{R}_{f}=0.43$ (2:1 hexanes/EtOAc); $\mathrm{mp}=139-140{ }^{\circ} \mathrm{C} ;{ }^{1} \mathrm{H} \mathrm{NMR}\left(\mathrm{CDCl}_{3}, 400 \mathrm{MHz}\right) \delta$ 7.46-7.38 (m, 3H), 7.38-7.32 (m, $2 \mathrm{H}), 6.87$ (br s, 1H), 5.76 (br d, $1 \mathrm{H}, J=7.5 \mathrm{~Hz}), 4.78(\mathrm{ddd}, 1 \mathrm{H}, J=8.7,7.5,4.6 \mathrm{~Hz}), 4.49(\mathrm{~d}, 1 \mathrm{H}, J=10.8 \mathrm{~Hz}), 4.41$ $(\mathrm{d}, 1 \mathrm{H}, J=10.8 \mathrm{~Hz}), 3.86-3.70(\mathrm{~m}, 2 \mathrm{H}) \mathrm{ppm} ;{ }^{13} \mathrm{C} \mathrm{NMR}\left(\mathrm{CD}_{3} \mathrm{CN}, 100 \mathrm{MHz}\right) \delta 158.2\left(\mathrm{q}, J_{\mathrm{CF}}=36.6 \mathrm{~Hz}\right), 138.8,129.7$, $129.3,127.9,116.8\left(\mathrm{q}, J_{\mathrm{CF}}=288.4 \mathrm{~Hz}\right), 94.0,78.6,58.6,45.1 \mathrm{ppm}$; IR (thin film) v 3302, 1712, 1560, 1460, 1369, 1183, 1089, 1018, 981, $859 \mathrm{~cm}^{-1}$; HRMS $\left(\mathrm{ES}^{+}\right)$calcd for $\mathrm{C}_{12} \mathrm{H}_{12} \mathrm{Cl}_{3} \mathrm{~F}_{3} \mathrm{~N}_{2} \mathrm{O}_{4} \mathrm{~S} 441.9535$ found $464.9433\left(\mathrm{MNa}^{+}\right)$.

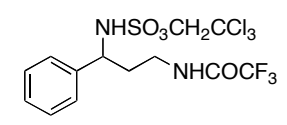

Purified by chromatography on silica gel (9:1 hexanes/EtOAc); pale yellow oil (70\%): TLC $\mathrm{R}_{f}=0.37(2: 1$ hexanes/EtOAc); ${ }^{1} \mathrm{H} \mathrm{NMR}\left(\mathrm{CDCl}_{3}, 400 \mathrm{MHz}\right) \delta 7.39-7.28(\mathrm{~m}, 5 \mathrm{H}), 7.14$ (br t, $\left.1 \mathrm{H}, J=5.9 \mathrm{~Hz}\right), 6.31(\mathrm{br} \mathrm{d}, 1 \mathrm{H}, J=$ $8.7 \mathrm{~Hz}), 4.53(\mathrm{td}, 1 \mathrm{H}, J=8.7,5.9 \mathrm{~Hz}), 4.32(\mathrm{~d}, 1 \mathrm{H}, J=10.8 \mathrm{~Hz}), 4.15(\mathrm{~d}, 1 \mathrm{H}, J=10.8 \mathrm{~Hz}), 3.67-3.57(\mathrm{~m}, 1 \mathrm{H}), 3.43-$ $3.30(\mathrm{~m}, 1 \mathrm{H}), 2.24-2.06(\mathrm{~m}, 2 \mathrm{H}) \mathrm{ppm} ;{ }^{13} \mathrm{C} \mathrm{NMR}\left(\mathrm{CDCl}_{3}, 100 \mathrm{MHz}\right) \delta 157.7$ (q, $\left.J_{\mathrm{CF}}=36.9 \mathrm{~Hz}\right), 139.5,129.2,128.7$, $126.4,115.6\left(\mathrm{q}, J_{\mathrm{CF}}=285.6 \mathrm{~Hz}\right), 92.9,77.9,57.0,37.0,35.3 \mathrm{ppm}$; IR (thin film) v 3294, 2952, 1709, 1560, 1496, 1458, 1368, 1213, 1181, 1087, 1048, 1017, 932, $858 \mathrm{~cm}^{-1}$; HRMS (ES ${ }^{+}$) calcd for $\mathrm{C}_{13} \mathrm{H}_{14} \mathrm{Cl}_{3} \mathrm{~F}_{3} \mathrm{~N}_{2} \mathrm{O}_{4} \mathrm{~S} 455.9692$ found $478.9585\left(\mathrm{MNa}^{+}\right)$.

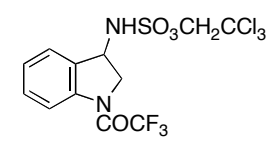

Purified by chromatography on silica gel (4:1 $\mathrm{CH}_{2} \mathrm{Cl}_{2} /$ hexanes); white solid (72\%): TLC $\mathrm{R}_{f}=0.40(4: 1$ hexanes/EtOAc); $\mathrm{mp}=119-121{ }^{\circ} \mathrm{C} ;{ }^{1} \mathrm{H} \mathrm{NMR}\left(\mathrm{CDCl}_{3}, 400 \mathrm{MHz}\right) \delta 8.21(\mathrm{~d}, 1 \mathrm{H}, J=8.2 \mathrm{~Hz}), 7.55,(\mathrm{ddt}, 1 \mathrm{H}, J=7.6$, $1.4,0.6 \mathrm{~Hz}), 7.45(\mathrm{tdd}, 1 \mathrm{H}, J=7.6,1.4,0.5 \mathrm{~Hz}), 7.29(\mathrm{td}, 1 \mathrm{H}, J=7.6,1.0 \mathrm{~Hz}), 5.34(\mathrm{td}, 1 \mathrm{H}, J=8.2,3.8 \mathrm{~Hz}), 5.24$ (br d, $1 \mathrm{H}, J=8.2 \mathrm{~Hz}), 4.72(\mathrm{~s}, 2 \mathrm{H}), 4.51(\mathrm{ddd}, 1 \mathrm{H}, J=12.5,7.8,0.6 \mathrm{~Hz}), 4.44-4.38(\mathrm{~m}, 1 \mathrm{H}) \mathrm{ppm} ;{ }^{13} \mathrm{C} \mathrm{NMR}\left(\mathrm{CDCl}{ }_{3}\right.$, $100 \mathrm{MHz}) \delta 154.3\left(\mathrm{q}, J_{\mathrm{CF}}=38.2 \mathrm{~Hz}\right), 141.2,131.0,128.6,126.8,125.6,118.1,115.7\left(\mathrm{q}, J_{\mathrm{CF}}=287.6 \mathrm{~Hz}\right), 93.2,78.1$, $54.9\left(\mathrm{q}, J_{\mathrm{CF}}=3.8 \mathrm{~Hz}\right), 54.6 \mathrm{ppm}$; IR (thin film) v 3383, 1690, 1602, 1487, 1440, 1373, 1256, 1186, 1151, 1087, 1047, 1014, 980, 856, $761 \mathrm{~cm}^{-1}$; HRMS $\left(\mathrm{ES}^{+}\right)$calcd for $\mathrm{C}_{12} \mathrm{H}_{10} \mathrm{Cl}_{3} \mathrm{~F}_{3} \mathrm{~N}_{2} \mathrm{O}_{4} \mathrm{~S} 439.9379$ found $462.9274\left(\mathrm{MNa}^{+}\right)$. 


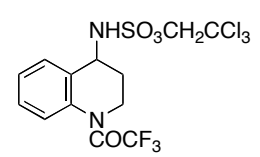

Purified by chromatography on silica gel (gradient elution: $13: 1 \rightarrow 4: 1$ hexanes/EtOAc); white solid (70\%): $\mathrm{TLC} \mathrm{R}_{f}=$ 0.34 (4:1 hexanes/EtOAc); mp = 130-132 ${ }^{\circ} \mathrm{C} ;{ }^{1} \mathrm{H}$ NMR $\left(\mathrm{CDCl}_{3}, 400 \mathrm{MHz}, 50{ }^{\circ} \mathrm{C}\right) \delta 7.73(\mathrm{br} \mathrm{d}, 1 \mathrm{H}, J=7.8 \mathrm{~Hz}), 7.57$ $(\mathrm{dd}, 1 \mathrm{H}, J=7.8,1.5 \mathrm{~Hz}), 7.36(\mathrm{td}, 1 \mathrm{H}, J=7.5,1.5 \mathrm{~Hz}), 7.30(\mathrm{dt}, 1 \mathrm{H}, J=7.5,1.5 \mathrm{~Hz}), 5.09(\mathrm{br} d, 1 \mathrm{H}, J=7.5 \mathrm{~Hz})$, $4.88(\mathrm{q}, 1 \mathrm{H}, J=6.8 \mathrm{~Hz}), 4.70(\mathrm{~d}, 1 \mathrm{H}, J=11.1 \mathrm{~Hz}), 4.69(\mathrm{~d}, 1 \mathrm{H}, J=11.1 \mathrm{~Hz}), 3.98-3.86(\mathrm{~m}, 2 \mathrm{H}), 2.50-2.41(\mathrm{~m}, 1 \mathrm{H})$, 2.38-2.30 (m, 1H) ppm; ${ }^{13} \mathrm{C} \mathrm{NMR}\left(\mathrm{CDCl}_{3}, 100 \mathrm{MHz}, 50{ }^{\circ} \mathrm{C}\right) \delta 156.0\left(\mathrm{q}, 1 \mathrm{C}, J_{\mathrm{CF}}=37.4 \mathrm{~Hz}\right), 136.7,129.1,129.0$, 128.6, 127.3, 124.6, $116.5\left(\mathrm{q}, J_{\mathrm{CF}}=288.4 \mathrm{~Hz}\right), 93.5,78.3,50.9,42.6$ (q, $\left.J_{\mathrm{CF}}=3.4 \mathrm{~Hz}\right), 31.2 \mathrm{ppm}$; IR (thin film) $v$ $3267,1686,1495,1433,1366,1292,1267,1205,1182,1149,1085,997,948,855 \mathrm{~cm}^{-1}$; HRMS (ES ${ }^{+}$) calcd for $\mathrm{C}_{13} \mathrm{H}_{12} \mathrm{Cl}_{3} \mathrm{~F}_{3} \mathrm{~N}_{2} \mathrm{O}_{4} \mathrm{~S} 453.9535$ found $476.9416\left(\mathrm{MNa}^{+}\right)$.

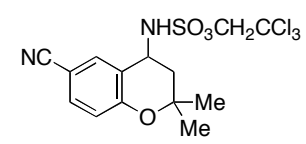

Purified by chromatography on silica gel (gradient elution: 9:1 $\rightarrow 4: 1$ hexanes/EtOAc); white solid (57\%): TLC $\mathrm{R}_{f}=$ 0.52 (2:1 hexanes/EtOAc); $\mathrm{mp}=179-181{ }^{\circ} \mathrm{C} ;{ }^{1} \mathrm{H} \mathrm{NMR}\left(\mathrm{CDCl}_{3}, 400 \mathrm{MHz}\right) \delta 7.90(\mathrm{dd}, 1 \mathrm{H}, J=2.0,1.1 \mathrm{~Hz}), 7.48$ $(\mathrm{dd}, 1 \mathrm{H}, J=8.3,2.0 \mathrm{~Hz}), 6.86(\mathrm{~d}, 1 \mathrm{H}, J=8.3 \mathrm{~Hz}), 4.90(\mathrm{br} \mathrm{d}, 1 \mathrm{H}, J=10.1 \mathrm{~Hz}), 4.82(\mathrm{td}, 1 \mathrm{H}, J=10.1,6.0 \mathrm{~Hz}), 4.78$ $(\mathrm{d}, 1 \mathrm{H}, J=10.8 \mathrm{~Hz}), 4.74(\mathrm{~d}, 1 \mathrm{H}, J=10.8 \mathrm{~Hz}), 2.47(\mathrm{dd}, 1 \mathrm{H}, J=13.6,6.0 \mathrm{~Hz}), 1.92(\mathrm{dd}, 1 \mathrm{H}, J=13.6,10.1 \mathrm{~Hz})$, $1.48(\mathrm{~s}, 3 \mathrm{H}), 1.33(\mathrm{~s}, 3 \mathrm{H}) \mathrm{ppm} ;{ }^{13} \mathrm{C}$ NMR $\left(\mathrm{d}_{6}\right.$-acetone, $\left.100 \mathrm{MHz}\right) \delta 210.0,158.4,133.8,133.5,123.3,119.5,104.2$, 94.8, 78.7, 77.8, 69.2, 48.3, 40.1, 24.6 ppm; IR (thin film) v 3169, 2980, 2229, 1611, 1489, 1372, 1302, 1269, 1182, 1129, 1084, 1005, 909, $847 \mathrm{~cm}^{-1}$; HRMS $\left(\mathrm{ES}^{+}\right)$calcd for $\mathrm{C}_{14} \mathrm{H}_{15} \mathrm{Cl}_{3} \mathrm{~N}_{2} \mathrm{O}_{4} \mathrm{~S} 411.9818$ found $434.9727\left(\mathrm{MNa}^{+}\right)$.

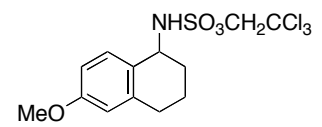

Purified by chromatography on silica gel (gradient elution: $13: 1 \rightarrow 4: 1$ hexanes/EtOAc); white solid (51\%, 7:1 C1/C4 mixture): $\mathrm{TLC} \mathrm{R}_{f}=0.36_{\mathrm{C} 1}, 0.38_{\mathrm{C} 4}\left(4: 1\right.$ hexanes/EtOAc); $\mathrm{C} 1$ isomer: $\mathrm{mp}=120-125{ }^{\circ} \mathrm{C} ;{ }^{1} \mathrm{H} \mathrm{NMR}\left(\mathrm{CDCl}{ }_{3}, 400 \mathrm{MHz}\right)$ $\delta 7.39(\mathrm{~d}, 1 \mathrm{H}, J=8.5 \mathrm{~Hz}), 6.77(\mathrm{dd}, 1 \mathrm{H}, J=8.6,2.7 \mathrm{~Hz}), 6.61(\mathrm{~d}, 1 \mathrm{H}, J=2.7 \mathrm{~Hz}), 4.84(\mathrm{br} \mathrm{d}, 1 \mathrm{H}, J=7.6 \mathrm{~Hz}), 4.74$ $(\mathrm{dt}, 1 \mathrm{H}, J=7.6,4.8 \mathrm{~Hz}), 4.68$ (s, 2H), 3.78 (s, 3H), 2.82-2.63 (m, 2H), 2.17-1.99 (m, 2H), 1.90-1.79 (m, 2H) ppm; ${ }^{13} \mathrm{C} \mathrm{NMR}\left(\mathrm{CDCl}_{3}, 100 \mathrm{MHz}\right) \delta$ 159.2, 139.1, 130.6, 126.6, 113.6, 113.0, 93.6, 78.0, 55.2, 53.0, 30.3, 29.1, 18.7 ppm; IR (thin film) $v$ 3305, 2943, 1609, 1579, 1503, 1447, 1432, 1363, 1277, 1257, 1235, 1183, 1076, 1027, 988, 946, $853 \mathrm{~cm}^{-1}$; HRMS $\left(\mathrm{ES}^{+}\right)$calcd for $\mathrm{C}_{13} \mathrm{H}_{16} \mathrm{Cl}_{3} \mathrm{NO}_{4} \mathrm{~S} 386.9866$ found $409.9760\left(\mathrm{MNa}^{+}\right)$.

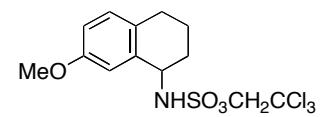

C4 isomer: $\mathrm{mp}=92-96{ }^{\circ} \mathrm{C} ;{ }^{1} \mathrm{H}$ NMR $\left(\mathrm{CDCl}_{3}, 400 \mathrm{MHz}\right) \delta 7.02(\mathrm{~d}, 1 \mathrm{H}, J=8.5 \mathrm{~Hz}), 6.99(\mathrm{~d}, 1 \mathrm{H}, J=2.6 \mathrm{~Hz}), 6.80$ $(\mathrm{dd}, 1 \mathrm{H}, J=8.5,2.6 \mathrm{~Hz}), 4.85$ (br d, 1H, $J=7.6 \mathrm{~Hz}), 4.75(\mathrm{dt}, 1 \mathrm{H}, J=7.6,5.2 \mathrm{~Hz}), 4.70(\mathrm{~s}, 2 \mathrm{H}), 3.79$ (s, 3H), 2.80$2.61(\mathrm{~m}, 2 \mathrm{H}), 2.14-2.02(\mathrm{~m}, 2 \mathrm{H}), 1.89-1.81(\mathrm{~m}, 2 \mathrm{H}) \mathrm{ppm} ;{ }^{13} \mathrm{C} \mathrm{NMR}\left(\mathrm{CDCl}_{3}, 100 \mathrm{MHz}\right) \delta 158.2,135.2,130.4,129.6$, 115.4, 112.9, 93.6, 78.1, 55.4, 53.7, 30.2, 28.0, 19.1 ppm; IR (thin film) $v$ 3308, 2935, 2860, 1612, 1503, 1448, 1363, 1252, 1234, 1182, 1078, 1034, 991, 946, $852 \mathrm{~cm}^{-1}$; HRMS (ES ${ }^{+}$) calcd for $\mathrm{C}_{13} \mathrm{H}_{16} \mathrm{Cl}_{3} \mathrm{NO}_{4} \mathrm{~S} 386.9866$ found $409.9763\left(\mathrm{MNa}^{+}\right)$. 


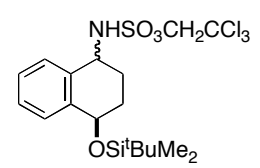

Purified by chromatography on silica gel (gradient elution: hexanes $\rightarrow 9: 1$ hexanes/EtOAc); white solid (60\%, 1:1 cis/trans mixture): TLC $\mathrm{R}_{f}=0.44,0.52$ (4:1 hexanes/EtOAc); higher $\mathrm{R}_{f}$ diastereomer: ${ }^{1} \mathrm{H} \mathrm{NMR}\left(\mathrm{CDCl}_{3}, 400 \mathrm{MHz}\right) \delta$ 7.48-7.45 (m, 1H), 7.37-7.28 (m, 3H), 5.57 (br d, $1 \mathrm{H}, J=8.5 \mathrm{~Hz}), 4.78(\mathrm{t}, 1 \mathrm{H}, J=5.4 \mathrm{~Hz}), 4.74(\mathrm{dt}, 1 \mathrm{H}, J=8.5,4.8$ $\mathrm{Hz}), 4.63(\mathrm{~d}, 1 \mathrm{H}, J=10.7 \mathrm{~Hz}), 4.54(\mathrm{~d}, 1 \mathrm{H}, J=10.7 \mathrm{~Hz}), 2.38-2.30(\mathrm{~m}, 1 \mathrm{H}), 2.07(\mathrm{ddd}, 1 \mathrm{H}, J=14.2,7.1,4.8 \mathrm{~Hz})$, 2.05-1.97 (m, 2H), $0.93(\mathrm{~s}, 9 \mathrm{H}), 0.18(\mathrm{~s}, 3 \mathrm{H}), 0.14(\mathrm{~s}, 3 \mathrm{H}) \mathrm{ppm} ;{ }^{13} \mathrm{C} \mathrm{NMR}\left(\mathrm{CDCl}_{3}, 100 \mathrm{MHz}\right) \delta 140.0,135.0,128.8$, 128.6, 128.3, 128.0, 93.5, 77.9, 69.0, 53.2, 28.7, 26.6, 25.8, 18.1, -4.3, -4.6 ppm; IR (thin film) v 3308, 2954, 2930, 2857, 1454, 1363, 1256, 1183, 1125, 1080, 1048, 1019, 995, 939, $838 \mathrm{~cm}^{-1}$; HRMS (ES ${ }^{+}$calcd for $\mathrm{C}_{18} \mathrm{H}_{28} \mathrm{Cl}_{3} \mathrm{NO}_{4} \mathrm{SSi}$ 487.0574 found $510.0476\left(\mathrm{MNa}^{+}\right)$; lower $\mathrm{R}_{f}$ diastereomer: ${ }^{1} \mathrm{H} \mathrm{NMR}\left(\mathrm{C}_{6} \mathrm{D}_{6}, 400 \mathrm{MHz}\right) \delta 7.38(\mathrm{~d}, 1 \mathrm{H}, J=7.7 \mathrm{~Hz})$, $7.35(\mathrm{~d}, 1 \mathrm{H}, J=7.7 \mathrm{~Hz}), 7.08(\mathrm{td}, 1 \mathrm{H}, J=7.8,1.4 \mathrm{~Hz}), 7.00(\mathrm{td}, 1 \mathrm{H}, J=7.8,1.4 \mathrm{~Hz}), 4.65-4.59(\mathrm{~m}, 1 \mathrm{H}), 4.58-4.50$ $(\mathrm{m}, 1 \mathrm{H}), 4.36(\mathrm{~d}, 1 \mathrm{H}, J=11.0 \mathrm{~Hz}), 4.32(\mathrm{~d}, 1 \mathrm{H}, J=11.0 \mathrm{~Hz}), 3.95(\mathrm{br} \mathrm{d}, 1 \mathrm{H}, J=8.0 \mathrm{~Hz}), 2.22(\mathrm{ddd}, 1 \mathrm{H}, J=10.5$, 8.7, $5.3 \mathrm{~Hz}$ ), 1.64 (ddd, 1H, $J=10.5,8.0,4.5 \mathrm{~Hz}), 1.52-1.48(\mathrm{~m}, 2 \mathrm{H}), 0.95$ (s, 9H), 0.09 (s, 3H), 0.06 (s, 3H) ppm; ${ }^{13} \mathrm{C} \mathrm{NMR}\left(\mathrm{CDCl}_{3}, 100 \mathrm{MHz}\right) \delta 140.3,134.2,128.43,128.42,128.2,128.0,93.5,78.0,68.1,53.6,29.8,27.2,25.8$, 18.1, -4.2, -4.6 ppm; IR (thin film) v 3292, 2956, 2930, 2857, 1454, 1363, 1255, 1180, 1127, 1085, 1051, 995, 926, $861,837 \mathrm{~cm}^{-1}$; HRMS (ES $)$ calcd for $\mathrm{C}_{18} \mathrm{H}_{28} \mathrm{Cl}_{3} \mathrm{NO}_{4} \mathrm{SSi} 487.0574$ found $510.0479\left(\mathrm{MNa}^{+}\right)$.

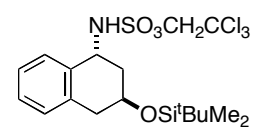

Purified by chromatography on silica gel (gradient elution: hexanes $\rightarrow 9: 1$ hexanes/EtOAc); white solid (53\%, 1:6 cis/trans mixture): TLC $\mathrm{R}_{f}=0.46_{\text {trans }}, 0.51_{\text {cis }}$ (4:1 hexanes/EtOAc); trans isomer: ${ }^{1} \mathrm{H} \mathrm{NMR}\left(\mathrm{CDCl}_{3}, 400 \mathrm{MHz}\right) \delta$ 7.54-7.50 (m, 1H), 7.27-7.22 (m, 2H), 7.12-7.08 (m, 1H), 5.00 (dt, 1H, J = 7.9, $5.6 \mathrm{~Hz}), 4.82(\mathrm{br} \mathrm{d}, 1 \mathrm{H}, J=7.9 \mathrm{~Hz})$, $4.70(\mathrm{~s}, 2 \mathrm{H}), 4.31-4.24(\mathrm{~m}, 1 \mathrm{H}), 3.04(\mathrm{dd}, 1 \mathrm{H}, J=16.6,4.6 \mathrm{~Hz}), 2.74(\mathrm{dd}, 1 \mathrm{H}, J=16.6,6.9 \mathrm{~Hz}), 2.21-2.17$ (m, $2 \mathrm{H})$, 0.88 (s, 9H), 0.12 (s, 3H), 0.10 (s, 3H) ppm; ${ }^{13} \mathrm{C} \mathrm{NMR}\left(\mathrm{CDCl}_{3}, 100 \mathrm{MHz}\right) \delta 135.3,133.9,129.7,128.3,128.2,126.8$, 93.5, 78.1, 64.7, 53.0, 38.9, 38.5, 25.8, 18.1, 1.0, -4.7 ppm; IR (thin film) v 3304, 2954, 2930, 2889, 2857, 1452, 1361, 1255, 1184, 1120, 1088, 1047, 1017, 990, 905, $837 \mathrm{~cm}^{-1}$; HRMS $\left(\mathrm{ES}^{+}\right)$calcd for $\mathrm{C}_{18} \mathrm{H}_{28} \mathrm{Cl}_{3} \mathrm{NO}_{4} \mathrm{SSi}_{487.0574}$ found $510.0467\left(\mathrm{MNa}^{+}\right)$.

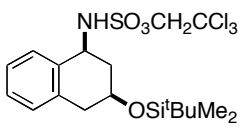

Cis isomer: ${ }^{1} \mathrm{H}$ NMR $\left(\mathrm{CDCl}_{3}, 400 \mathrm{MHz}\right) \delta$ 7.54-7.48 (m, 1H), 7.28-7.22 (m, 2H), 7.12-7.04 (m, $\left.1 \mathrm{H}\right), 6.55(\mathrm{br} \mathrm{d}, 1 \mathrm{H}$, $J=9.4 \mathrm{~Hz}), 4.81(\mathrm{ddd}, 1 \mathrm{H}, J=9.4,5.3,2.7 \mathrm{~Hz}), 4.63(\mathrm{~d}, 1 \mathrm{H}, J=10.7 \mathrm{~Hz}), 4.60(\mathrm{~d}, 1 \mathrm{H}, J=10.7 \mathrm{~Hz}), 4.54-4.50$ (m, 1H), 2.98 (dd, 1H, $J=17.0,3.8 \mathrm{~Hz}$ ), 2.74 (br d, 1H, $J=17.0 \mathrm{~Hz}$ ), 2.49 (dddd, 1H, $J=14.3,4.7,2.7,1.9 \mathrm{~Hz}), 2.10$ (ddd, $1 \mathrm{H}, J=14.3,5.3,1.9 \mathrm{~Hz}), 0.88(\mathrm{~s}, 9 \mathrm{H}), 0.16(\mathrm{~s}, 3 \mathrm{H}), 0.11(\mathrm{~s}, 3 \mathrm{H}) \mathrm{ppm} ;{ }^{13} \mathrm{C} \mathrm{NMR}\left(\mathrm{CDCl}_{3}, 100 \mathrm{MHz}\right) \delta 134.3$, $132.8,130.1,129.5,128.3,126.8,93.7,77.8,66.8,51.5,37.3,34.9,25.8,18.0,-4.9,-5.0$ ppm; IR (thin film) v 3304, 2954, 2930, 2896, 2857, 1456, 1362, 1255, 1184, 1120, 1089, 1047, 1016, 990, $837 \mathrm{~cm}^{-1}$; HRMS (ES HRalcd for $^{+}$ $\mathrm{C}_{18} \mathrm{H}_{28} \mathrm{Cl}_{3} \mathrm{NO}_{4} \mathrm{SSi} 487.0574$ found $510.0475\left(\mathrm{MNa}^{+}\right)$.

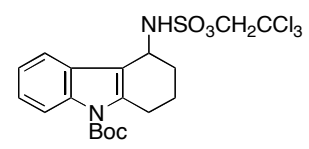

Purified by chromatography on silica gel (gradient elution: $13: 1 \rightarrow 4: 1$ hexanes/EtOAc); white solid (50\%, 8:1 C4/C1 mixure): $\mathrm{TLC} \mathrm{R}_{f}=0.44_{\mathrm{C} 4}, 0.52_{\mathrm{C} 1}\left(4: 1\right.$ hexanes/EtOAc); $\mathrm{C} 4$ isomer: $\mathrm{mp}=143-146{ }^{\circ} \mathrm{C} ;{ }^{1} \mathrm{H} \mathrm{NMR}\left(\mathrm{CDCl}_{3}, 400 \mathrm{MHz}\right.$, 
$\left.40{ }^{\circ} \mathrm{C}\right) \delta 8.13(\mathrm{ddd}, 1 \mathrm{H}, J=7.7,1.3,0.7 \mathrm{~Hz}), 7.76(\mathrm{ddd}, 1 \mathrm{H}, J=7.7,1.6,0.7 \mathrm{~Hz}), 7.30(\mathrm{td}, 1 \mathrm{H}, J=7.7,1.6 \mathrm{~Hz}), 7.25$ (td, $1 \mathrm{H}, J=7.7,1.3 \mathrm{~Hz}), 5.01$ (ddd, $1 \mathrm{H}, J=6.0,5.0,4.4 \mathrm{~Hz}), 4.75$ (d, $1 \mathrm{H}, J=11.0 \mathrm{~Hz}), 4.75$ (br d, $1 \mathrm{H}, J=6.0 \mathrm{~Hz}$ ), $4.74(\mathrm{~d}, 1 \mathrm{H}, J=11.0 \mathrm{~Hz}), 3.18(\mathrm{dt}, 1 \mathrm{H}, J=18.4,4.4 \mathrm{~Hz}), 2.95-2.84(\mathrm{~m}, 1 \mathrm{H}), 2.34-2.28(\mathrm{~m}, 1 \mathrm{H}), 2.13-1.90(\mathrm{~m}, 3 \mathrm{H})$, 1.68 (s, 9H) ppm; ${ }^{13} \mathrm{C} \mathrm{NMR}\left(\mathrm{CDCl}_{3}, 100 \mathrm{MHz}\right) \delta 150.2,139.1,135.7,127.3,124.2,123.1,118.1,115.4,113.8,93.6$, 84.2, 77.9, 48.1, 29.1, 28.2, 25.5, 18.5 ppm; IR (thin film) v 3280, 2979, 2947, 1732, 1610, 1458, 1430, 1396, 1371, 1325, 1258, 1224, 1180, 1161, 1142, 1118, 1076, 1021, 1001, 964, 911, $849 \mathrm{~cm}^{-1}$; HRMS (ES ${ }^{+}$) calcd for $\mathrm{C}_{19} \mathrm{H}_{23} \mathrm{Cl}_{3} \mathrm{~N}_{2} \mathrm{O}_{5} \mathrm{~S} 496.0393$ found $519.0290\left(\mathrm{MNa}^{+}\right)$.

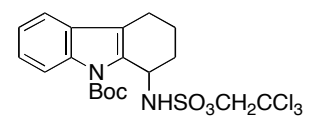

$\mathrm{C} 1$ isomer: $\mathrm{mp}=116-120{ }^{\circ} \mathrm{C} ;{ }^{1} \mathrm{H}$ NMR $\left(\mathrm{CDCl}_{3}, 400 \mathrm{MHz}\right) \delta 8.01(\mathrm{dt}, 1 \mathrm{H}, J=8.4,0.8 \mathrm{~Hz}), 7.47(\mathrm{ddd}, 1 \mathrm{H}, J=7.2$, $1.2,0.8 \mathrm{~Hz}), 7.34(\mathrm{ddd}, 1 \mathrm{H}, J=8.4,7.2,1.2 \mathrm{~Hz}), 7.24(\mathrm{ddd}, 1 \mathrm{H}, J=8.4,7.2,1.2 \mathrm{~Hz}), 5.26(\mathrm{~m}, 2 \mathrm{H}), 4.85(\mathrm{~d}, 1 \mathrm{H}, J=$ $10.7 \mathrm{~Hz}), 4.71(\mathrm{~d}, 1 \mathrm{H}, J=10.7 \mathrm{~Hz}), 2.85(\mathrm{ddd}, 1 \mathrm{H}, J=16.9,5.3,2.2 \mathrm{~Hz}), 2.77-2.70(\mathrm{~m}, 1 \mathrm{H}), 2.60(\mathrm{ddd}, 1 \mathrm{H}, J=$ 16.9, 10.4, $6.7 \mathrm{~Hz}), 2.14-1.93(\mathrm{~m}, 2 \mathrm{H}), 1.88-1.79(\mathrm{~m}, 1 \mathrm{H}), 1.72(\mathrm{~s}, 9 \mathrm{H}) \mathrm{ppm} ;{ }^{13} \mathrm{C} \mathrm{NMR}\left(\mathrm{CDCl}_{3}, 100 \mathrm{MHz}\right) \delta 150.5$, 135.8, 131.1, 128.6, 125.4, 122.9, 121.8, 118.9, 115.8, 93.6, 85.0, 78.3, 49.5, 28.3, 28.2, 20.9, 16.8 ppm; IR (thin film) $v 3309,2934,1728,1455,1370,1313,1256,1222,1175,1141,1119,1087,1022,1002,964,911,853 \mathrm{~cm}^{-1}$.

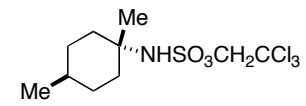

Purified by chromatography on silica gel (gradient elution: 13:1 $\rightarrow 4: 1$ hexanes/EtOAc); white solid (46\%): TLC $\mathrm{R}_{f}=$ 0.44 (4:1 hexanes/EtOAc); mp = 89-91 ${ }^{\circ} \mathrm{C} ;{ }^{1} \mathrm{H}$ NMR $\left(\mathrm{CDCl}_{3}, 400 \mathrm{MHz}\right) \delta 4.78$ (br s, 1H), $4.60(\mathrm{~s}, 2 \mathrm{H}), 1.92-1.85$ $(\mathrm{m}, 2 \mathrm{H}), 1.70-1.60(\mathrm{~m}, 4 \mathrm{H}), 1.46-1.36(\mathrm{~m}, 1 \mathrm{H}), 1.42(\mathrm{~s}, 3 \mathrm{H}), 1.16-1.04(\mathrm{~m}, 2 \mathrm{H}), 0.90(\mathrm{~d}, 3 \mathrm{H}, J=6.6 \mathrm{~Hz}) \mathrm{ppm} ;{ }^{13} \mathrm{C}$ NMR $\left(\mathrm{CDCl}_{3}, 100 \mathrm{MHz}\right) \delta 93.4,78.1,58.5,37.5,31.5,30.8,22.8,21.6 \mathrm{ppm}$; IR (thin film) v 3296, 2928, 2861, 1448, 1357, 1186, 1175, 1126, 1090, 1045, 1018, 984, $851 \mathrm{~cm}^{-1}$; HRMS $\left(\mathrm{ES}^{+}\right)$calcd for $\mathrm{C}_{10} \mathrm{H}_{18} \mathrm{Cl}_{3} \mathrm{NO}_{3} \mathrm{~S} 337.0073$ found $359.9985\left(\mathrm{MNa}^{+}\right)$.

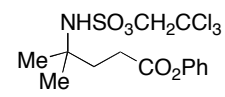

Purified by chromatography on silica gel (gradient elution: $9: 1 \rightarrow 4: 1$ hexanes/EtOAc); pale yellow solid (25\%): TLC $\mathrm{R}_{f}=0.52\left(2: 1\right.$ hexanes/EtOAc); $\mathrm{mp}=70-75{ }^{\circ} \mathrm{C} ;{ }^{1} \mathrm{H}$ NMR $\left(\mathrm{CDCl}_{3}, 400 \mathrm{MHz}\right) \delta$ 7.41-7.36 (m, 2H), 7.26-7.22 (m, 1H), 7.10-7.06 (m, 2H), 5.08 (br s, 1H), $4.64(\mathrm{~s}, 2 \mathrm{H}), 2.73(\mathrm{t}, 2 \mathrm{H}, J=7.6 \mathrm{~Hz}), 2.09$ (t, 2H, $J=7.6 \mathrm{~Hz}), 1.47(\mathrm{~s}, 6 \mathrm{H})$ ppm; ${ }^{13} \mathrm{C}$ NMR $\left(\mathrm{CDCl}_{3}, 100 \mathrm{MHz}\right) \delta$ 172.3, 150.5, 129.5, 126.0, 121.4, 93.4, 78.0, 57.5, 36.5, 29.3, $27.3 \mathrm{ppm}$; IR (thin film) v 3295, 2976, 1751, 1594, 1493, 1406, 1389, 1374, 1268, 1193, 1165, 1087, 1046, 1004, 957, $855 \mathrm{~cm}^{-1}$; HRMS (ES ${ }^{+}$) calcd for $\mathrm{C}_{14} \mathrm{H}_{18} \mathrm{Cl}_{3} \mathrm{NO}_{5} \mathrm{~S} 416.9971$ found $439.9864\left(\mathrm{MNa}^{+}\right)$.

\section{$\mathrm{Me} \mathrm{NHSO}_{3} \mathrm{CH}_{2} \mathrm{CCl}_{3}$}

Purified by chromatography on silica gel (gradient elution: $\mathrm{CH}_{2} \mathrm{Cl}_{2} \rightarrow 10: 1 \mathrm{CH}_{2} \mathrm{Cl}_{2} / \mathrm{EtOAc}$ ); colorless oil (10\%): TLC $\mathrm{R}_{f}=0.46\left(2: 1\right.$ hexanes/EtOAc); ${ }^{1} \mathrm{H}$ NMR $\left(\mathrm{CDCl}_{3}, 400 \mathrm{MHz}\right) \delta$ 7.49-7.46 (m, 2H), 7.43-7.38 (m, 2H), 7.36-7.31 (m, $1 \mathrm{H}), 5.59(\mathrm{br} \mathrm{s}, 1 \mathrm{H}), 4.56(\mathrm{~d}, 1 \mathrm{H}, J=10.8 \mathrm{~Hz}), 4.54(\mathrm{~d}, 1 \mathrm{H}, J=10.8 \mathrm{~Hz}), 4.50(\mathrm{~d}, 1 \mathrm{H}, J=11.7 \mathrm{~Hz}), 4.24(\mathrm{~d}, 1 \mathrm{H}, J=$ $11.7 \mathrm{~Hz}), 2.08(\mathrm{~s}, 3 \mathrm{H}), 1.88$ (s, 3H) ppm; ${ }^{13} \mathrm{C} \mathrm{NMR}\left(\mathrm{CDCl}_{3}, 100 \mathrm{MHz}\right) \delta 170.8,140.6,128.8,128.2,125.8,93.3$, 78.1, 70.2, 61.9, 22.7, 20.8 ppm; IR (thin film) v 3289, 1743, 1446, 1374, 1239, 1185, 1087, 1045, 1029, 997, 928, $857 \mathrm{~cm}^{-1}$; HRMS (ES ${ }^{+}$) calcd for $\mathrm{C}_{13} \mathrm{H}_{16} \mathrm{Cl}_{3} \mathrm{NO}_{5} \mathrm{~S} 402.9815$ found $425.9710\left(\mathrm{MNa}^{+}\right)$. 
$\overbrace{}^{\mathrm{NHSO}_{3} \mathrm{CH}_{2} \mathrm{CCl}_{3}}$

Reaction performed with $1.8 \mathrm{mmol}$ (1 equiv) of cyclohexane. Purified by chromatography on silica gel (19:1 hexanes/EtOAc); white solid (38\%): $\mathrm{TLC} \mathrm{R}=0.52$ (4:1 hexanes/EtOAc); mp = 79-81 ${ }^{\circ} \mathrm{C} ;{ }^{1} \mathrm{H} \mathrm{NMR}\left(\mathrm{CDCl}_{3}, 400\right.$ $\mathrm{MHz}) \delta 4.62(\mathrm{~s}, 2 \mathrm{H}), 4.46$ (br d, 1H, $J=7.3 \mathrm{~Hz}), 3.49-3.39(\mathrm{~m}, 1 \mathrm{H}), 2.12-2.04(\mathrm{~m}, 2 \mathrm{H}), 1.79-1.71(\mathrm{~m}, 2 \mathrm{H}), 1.65-$ $1.56(\mathrm{~m}, 1 \mathrm{H}), 1.41-1.12(\mathrm{~m}, 5 \mathrm{H}) \mathrm{ppm} ;{ }^{13} \mathrm{C} \mathrm{NMR}\left(\mathrm{CDCl}_{3}, 100 \mathrm{MHz}\right) \delta 93.5,78.1,54.2,33.6,25.0,24.6 \mathrm{ppm}$; IR $\left(\right.$ thin film) $v$ 3305, 2932, 2857, 1452, 1360, 1182, 1080, 1048, 1022, 998, 933, 890, 855, $757 \mathrm{~cm}^{-1}$; $\left.\mathrm{HRMS}_{(\mathrm{ES}}{ }^{+}\right)$ calcd for $\mathrm{C}_{8} \mathrm{H}_{14} \mathrm{Cl}_{3} \mathrm{NO}_{3} \mathrm{~S} 308.9760$ found $331.9663\left(\mathrm{MNa}^{+}\right)$.

$\overbrace{\mathrm{Ph}}^{\mathrm{Me}} \overbrace{\mathrm{NHSO}}^{\mathrm{NH}_{3} \mathrm{CH}_{2} \mathrm{CCl}_{3}}$

Purified by chromatography on silica gel (19:1 hexanes/EtOAc); white solid (70\%): TLC $\mathrm{R}_{f}=0.50$ (4:1 hexanes/EtOAc); $\mathrm{mp}=71-73{ }^{\circ} \mathrm{C} ;{ }^{1} \mathrm{H} \mathrm{NMR}\left(\mathrm{CDCl}_{3}, 400 \mathrm{MHz}\right) \delta$ 7.39-7.34 (m, 2H), 7.32-7.28 (m, 3H), 5.35 (br d, $1 \mathrm{H}, J=7.8 \mathrm{~Hz}), 4.56(\mathrm{q}, 1 \mathrm{H}, J=7.8 \mathrm{~Hz}), 4.28(\mathrm{~d}, 1 \mathrm{H}, J=10.8 \mathrm{~Hz}), 4.20(\mathrm{~d}, 1 \mathrm{H}, J=10.8 \mathrm{~Hz}), 1.80(\mathrm{dt}, 1 \mathrm{H}, J=13.7$, $7.5 \mathrm{~Hz}), 1.66(\mathrm{dt}, 1 \mathrm{H}, J=13.7,7.0 \mathrm{~Hz}), 1.56(\mathrm{sept}, 1 \mathrm{H}, J=6.7 \mathrm{~Hz}), 0.94(\mathrm{~d}, 6 \mathrm{H}, J=6.6 \mathrm{~Hz}) \mathrm{ppm} ;{ }^{13} \mathrm{C} \mathrm{NMR}\left(\mathrm{CDCl}{ }_{3}\right.$, $100 \mathrm{MHz}) \delta 140.9,129.0,128.2,126.6,93.2,77.9,57.8,46.1,24.7,22.4,22.2 \mathrm{ppm}$; IR (thin film) v 3308, 2959, 1496, 1457, 1427, 1366, 1270, 1181, 1137, 1087, 1050, 1005, 971, 941, 855, $757 \mathrm{~cm}^{-1}$; HRMS (ES ${ }^{+}$) calcd for $\mathrm{C}_{13} \mathrm{H}_{18} \mathrm{Cl}_{3} \mathrm{NO}_{3} \mathrm{~S} 373.0073$ found $395.9977\left(\mathrm{MNa}^{+}\right)$.

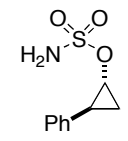

Purified by chromatography on silica gel (4:1 hexanes/EtOAc); white solid (75\%): $\mathrm{TLC}_{f}=0.32(2: 1$ hexanes/EtOAc); $\mathrm{mp}=89-92{ }^{\circ} \mathrm{C} ;{ }^{1} \mathrm{H} \mathrm{NMR}\left(\mathrm{CDCl}_{3}, 400 \mathrm{MHz}\right) \delta$ 7.32-7.27 (m, 2H), 7.25-7.20 (m, 1H), 7.12-7.09 (m, 2H), 4.82 (br s, 2H), 4.18 (ddd, 1H, $J=6.7,3.3,2.6 \mathrm{~Hz}), 2.55$ (ddd, 1H, $J=10.7,7.3,2.6 \mathrm{~Hz}$ ), 1.60 (ddd, 1H, $J$ $=10.7,7.0,3.3 \mathrm{~Hz}), 1.29$ (q, $1 \mathrm{H}, J=7.0 \mathrm{~Hz}) \mathrm{ppm} ;{ }^{13} \mathrm{C} \mathrm{NMR}\left(\mathrm{CDCl}_{3}, 100 \mathrm{MHz}\right) \delta 138.3,128.6,126.7,126.5,60.6$, 22.7, 13.8 ppm; IR (thin film) v 3379, 3281, 1355, 1176, 1130, 1049, 976, 949, 923, 844, $790 \mathrm{~cm}^{-1}$; $\mathrm{HRMS}^{\left(\mathrm{ES}^{+}\right)}$ calcd for $\mathrm{C}_{9} \mathrm{H}_{11} \mathrm{NO}_{3} \mathrm{~S} 213.0460$ found $236.0361\left(\mathrm{MNa}^{+}\right)$.

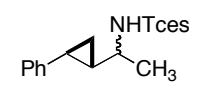

Purified by chromatography on silica gel (gradient elution: 11:1 $\rightarrow 4: 1$ hexanes/EtOAc); clear oil (78\%, 3:2 diastereomeric mixture): TLC $\mathrm{R}_{f}=0.47,0.53$ (4:1 hexanes/EtOAc); higher $\mathrm{R}_{f}$ diastereomer: ${ }^{1} \mathrm{H} \mathrm{NMR}\left(\mathrm{CDCl}_{3}, 400\right.$ $\mathrm{MHz}) \delta$ 7.28-7.24 (m, 2H), 7.19-7.14 (m, 1H), 7.08-7.04 (m. 2H), $4.71(\mathrm{br} \mathrm{d}, 1 \mathrm{H}, J=6.0 \mathrm{~Hz}), 4.61(\mathrm{~d}, 1 \mathrm{H}, J=10.8$ $\mathrm{Hz}), 4.58(\mathrm{~d}, 1 \mathrm{H}, J=10.8 \mathrm{~Hz}), 3.24-3.14(\mathrm{~m}, 1 \mathrm{H}), 2.09-2.04(\mathrm{~m}, 1 \mathrm{H}), 1.43(\mathrm{~d}, 3 \mathrm{H}, J=6.6 \mathrm{~Hz}), 1.29-1.21(\mathrm{~m}, 1 \mathrm{H})$, $1.05(\mathrm{dt}, 1 \mathrm{H}, J=8.7,5.3 \mathrm{~Hz}), 0.98(\mathrm{dt}, 1 \mathrm{H}, J=9.0,5.3 \mathrm{~Hz}) \mathrm{ppm} ;{ }^{13} \mathrm{C} \mathrm{NMR}\left(\mathrm{CDCl}_{3}, 100 \mathrm{MHz}\right) \delta 141.6,128.5$, 126.0, 125.7, 93.5, 78.0, 56.2, 29.1, 22.2, 20.9, 14.4 ppm; IR (thin film) v 3308, 2932, 1605, 1498, 1429, 1364, 1263, 1182, 1118, 1088, 1002, 853, $753 \mathrm{~cm}^{-1}$; HRMS $\left(\mathrm{ES}^{+}\right)$calcd for $\mathrm{C}_{13} \mathrm{H}_{16} \mathrm{Cl}_{3} \mathrm{NO}_{3} \mathrm{~S} 370.9916$ found 393.9808 $\left(\mathrm{MNa}^{+}\right)$; lower $\mathrm{R}_{f}$ diastereomer: ${ }^{1} \mathrm{H}$ NMR $\left(\mathrm{CDCl}_{3}, 400 \mathrm{MHz}\right) \delta$ 7.29-7.25 (m, 2H), 7.20-7.16 (m, 1H), 7.08-7.05 (m, 2H), 4.74 (br s, 1H), $4.65(\mathrm{~s}, 2 \mathrm{H}), 3.27-3.17(\mathrm{~m}, 1 \mathrm{H}), 1.89(\mathrm{dt}, 1 \mathrm{H}, J=9.3,4.7 \mathrm{~Hz}), 1.43(\mathrm{~d}, 3 \mathrm{H}, J=6.6 \mathrm{~Hz}), 1.28-$ $1.21(\mathrm{~m}, 1 \mathrm{H}), 1.17(\mathrm{dt}, 1 \mathrm{H}, J=8.9,5.4 \mathrm{~Hz}), 1.05(\mathrm{dt}, 1 \mathrm{H}, J=8.3,5.4 \mathrm{~Hz}) \mathrm{ppm} ;{ }^{13} \mathrm{C} \mathrm{NMR}\left(\mathrm{CDCl}_{3}, 100 \mathrm{MHz}\right) \delta$ $141.5,128.4,126.0,125.7,93.5,78.1,55.9,28.9,22.0,21.1,14.6$ ppm; IR (thin film) $v$ 3302, 2977, 2929, 1605, 1498, 1431, 1363, 1264, 1181, 1119, 1088, 1046, 1027, 998, 852, $753 \mathrm{~cm}^{-1}$; HRMS (ES ${ }^{+}$calcd for $\mathrm{C}_{13} \mathrm{H}_{16} \mathrm{Cl}_{3} \mathrm{NO}_{3} \mathrm{~S}$ 370.9916 found $393.9818\left(\mathrm{MNa}^{+}\right)$. 


\section{Experimental procedure and characterization data for ${ }^{15} \mathrm{~N}$-trichloroethoxysulfonamide and ${ }^{15} \mathrm{~N}$-amine:}

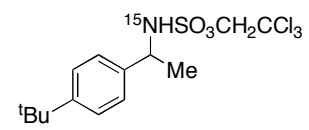

Purified by chromatography on silica gel (19:1 hexanes/EtOAc); white solid (80\%): TLC $\mathrm{R}_{f}=0.48$ (4:1 hexanes/EtOAc); ${ }^{1} \mathrm{H}$ NMR $\left(\mathrm{CDCl}_{3}, 400 \mathrm{MHz}\right) \delta$ 7.42-7.38 (m, 2H), 7.31-7.27 (m, 2H), $5.21(\mathrm{br} \mathrm{dd}, 1 \mathrm{H}, J=6.9 \mathrm{~Hz}$, $\left.J_{\mathrm{HN}}=87.4 \mathrm{~Hz}\right), 4.70$ (quint, $\left.1 \mathrm{H}, J=6.9 \mathrm{~Hz}\right), 4.33(\mathrm{~d}, 1 \mathrm{H}, J=10.8 \mathrm{~Hz}), 4.31(\mathrm{~d}, 1 \mathrm{H}, J=10.8 \mathrm{~Hz}) 1.62(\mathrm{dd}, 3 \mathrm{H}, J=6.9$ $\left.\mathrm{Hz}, J_{\mathrm{HN}}=3.5\right), 1.31(\mathrm{~s}, 9 \mathrm{H}) \mathrm{ppm} ;{ }^{13} \mathrm{C} \mathrm{NMR}\left(\mathrm{CDCl}_{3}, 100 \mathrm{MHz}\right) \delta 151.3,138.3,126.0,125.9,93.3,78.0,54.6\left(\mathrm{~d}, J_{\mathrm{CN}}\right.$ $=5.3 \mathrm{~Hz}$ ), 34.5, 31.3, 22.7 ppm; IR (thin film) v 3296, 2965,1429, 1365, 1269, 1181, 1114, 1086, 1013, 967, 848, 757, $726 \mathrm{~cm}^{-1}$; HRMS $\left(\mathrm{ES}^{+}\right)$calcd for $\mathrm{C}_{14} \mathrm{H}_{20} \mathrm{Cl}_{3}{ }^{15} \mathrm{NO}_{3} \mathrm{~S} 388.0199$ found $411.0096\left(\mathrm{MNa}^{+}\right)$.

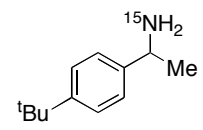

A solution of alkoxysulfonamide $(70 \mathrm{mg}, 0.18 \mathrm{mmol})$ in $3.0 \mathrm{~mL}$ of 1:1 $\mathrm{MeOH} / \mathrm{AcOH}$ was transferred via cannula into a flask containing $\mathrm{Zn}(\mathrm{Cu})$ couple $(118 \mathrm{mg}, 1.8 \mathrm{mmol}, 10.0$ equiv). Transfer of the starting material was made quantitative with an additional $2.0 \mathrm{~mL}$ of $1: 1 \mathrm{MeOH} / \mathrm{AcOH}$. The resulting suspension was stirred vigorously for 48 h. After this time, the reaction contents were filtered through a small pad of Celite and the filter cake was rinsed with $\mathrm{MeOH}$. The combined filtrates were concentrated under reduced pressure to afford a white solid, which was redissolved in $7.5 \mathrm{~mL}$ of anhydrous methanolic $\mathrm{HCl}$ (prepared with $0.56 \mathrm{~mL}$ of $\mathrm{CH}_{3} \mathrm{COCl}$ in $7.5 \mathrm{~mL} \mathrm{of} \mathrm{MeOH}$ ). The solution was heated to $40{ }^{\circ} \mathrm{C}$ for $12 \mathrm{~h}$ and then concentrated under reduced pressure. The resulting viscous oil was dissolved in $25 \mathrm{~mL}$ of EtOAc and transferred to a separatory funnel containing $5 \mathrm{~mL}$ of saturated aqueous $\mathrm{K}_{2} \mathrm{CO}_{3}$. The organic phase was collected and the aqueous layer was extracted with 2 x $25 \mathrm{~mL}$ of EtOAc. The combined organic extracts were dried over $\mathrm{MgSO}_{4}$, filtered, and concentrated under reduced pressure. Purification by chromatography on silica gel $\left(7: 1 \mathrm{CH}_{2} \mathrm{Cl}_{2} / \mathrm{MeOH}\right)$ afforded the desired amine as a white solid (87\%): TLC $\mathrm{R}_{f}=$ $0.33\left(6.7: 1 \mathrm{CH}_{2} \mathrm{Cl}_{2} / \mathrm{MeOH}\right) ;{ }^{1} \mathrm{H} \mathrm{NMR}\left(\mathrm{CDCl}_{3}, 400 \mathrm{MHz}\right) \delta 7.39$ (br s, 4H), 4.33 (q, $\left.1 \mathrm{H}, J=6.8 \mathrm{~Hz}\right), 1.60(\mathrm{dd}, 3 \mathrm{H}, J$

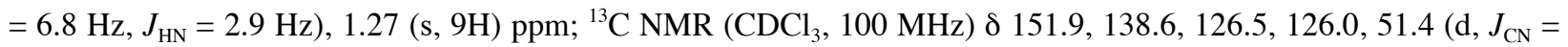
$4.8 \mathrm{~Hz}$ ), $22.7 \mathrm{ppm}$; IR (thin film) v 3279, 2796, 1608, 1508, 1459, 1362, 1320, 1231, 1111, 1076, $1021,831 \mathrm{~cm}^{-1}$; HRMS $\left(\mathrm{ES}^{+}\right)$calcd for $\mathrm{C}_{12} \mathrm{H}_{19}{ }^{15} \mathrm{~N} 178.1487$ found $179.1566\left(\mathrm{M}^{+}\right)$.

\section{Experimental procedure and characterization data for Hammett study}

A flask containing $\mathrm{H}_{2} \mathrm{NSO}_{3} \mathrm{CH}_{2} \mathrm{CCl}_{3}(27 \mathrm{mg}, 0.12 \mathrm{mmol})$ in $0.6 \mathrm{~mL}$ of $\mathrm{C}_{6} \mathrm{H}_{6}$ was charged with $\mathrm{Rh}{ }_{2}(\mathrm{esp})_{2}(9 \mathrm{mg}, 12$ $\mu \mathrm{mol}, 0.02$ equiv), ethylbenzene $(127 \mathrm{mg}, 1.2 \mathrm{mmol})$, and a $p$-substituted ethylbenzene substrate (1.2 mmol). To this bright green mixture was added $0.22 \mathrm{~mL}$ of a $0.83 \mathrm{M} \mathrm{C}_{6} \mathrm{H}_{6}$ solution of $\mathrm{PhI}\left(\mathrm{O}_{2} \mathrm{C}^{t} \mathrm{Bu}\right)_{2}(0.18$ mmol, 0.15 equiv $)$ via syringe pump over $3 \mathrm{~h}$. The color of the solution remained deep green throughout the addition of $\mathrm{PhI}\left(\mathrm{O}_{2} \mathrm{C}^{\mathrm{t}} \mathrm{Bu}\right)_{2}$. Following the transfer of oxidant, the solution was stirred at $23{ }^{\circ} \mathrm{C}$ for $1-2 \mathrm{~h}$ then concentrated under reduced pressure to an oily green residue. Product ratios were determined by ${ }^{1} \mathrm{H}$ NMR integration $(400 \mathrm{MHz})$ of the unpurified reaction mixture. Reactions were performed in triplicate and the average product ratio used to generate the Hammett plot.

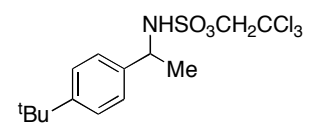

Purified by chromatography on silica gel (19:1 hexanes/EtOAc); white solid (78\%): TLC $\mathrm{R}_{f}=0.48$ (4:1 hexanes/EtOAc); $\mathrm{mp}=88-90{ }^{\circ} \mathrm{C} ;{ }^{1} \mathrm{H} \mathrm{NMR}\left(\mathrm{CDCl}_{3}, 400 \mathrm{MHz}\right) \delta$ 7.42-7.38 (m, 2H), 7.31-7.25 (m, $\left.2 \mathrm{H}\right), 4.80$ (br d, 
$1 \mathrm{H}, J=6.9 \mathrm{~Hz}$ ), 4.71 (quint, $1 \mathrm{H}, J=6.9 \mathrm{~Hz}), 4.37(\mathrm{~s}, 2 \mathrm{H}), 1.63(\mathrm{~d}, 3 \mathrm{H}, J=6.9 \mathrm{~Hz}), 1.31(\mathrm{~s}, 9 \mathrm{H}) \mathrm{ppm} ;{ }^{13} \mathrm{C} \mathrm{NMR}$ $\left(\mathrm{CDCl}_{3}, 100 \mathrm{MHz}\right) \delta 151.4,138.3,126.0,125.9,93.3,78.0,54.6,34.6,31.3,22.6 \mathrm{ppm}$; IR (thin film) v 3325, 2965, 2870, 1615, 1513, 1433, 1365, 1269, 1182, 1132, 1115, 1083, 1047, 1015, 977, 879, 851, $758 \mathrm{~cm}^{-1}$; $\mathrm{HRMS}_{\left(\mathrm{ES}^{+}\right)}$ calcd for $\mathrm{C}_{14} \mathrm{H}_{20} \mathrm{Cl}_{3} \mathrm{NO}_{3} \mathrm{~S} 387.0229$ found $410.0129\left(\mathrm{MNa}^{+}\right)$.

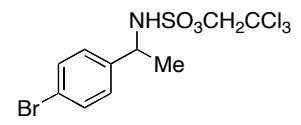

Purified by chromatography on silica gel (gradient elution: $11: 1 \rightarrow 4: 1$ hexanes/EtOAc); white solid (61\%): TLC $\mathrm{R}_{f}=$ 0.38 (4:1 hexanes/EtOAc); $\mathrm{mp}=55-57{ }^{\circ} \mathrm{C} ;{ }^{1} \mathrm{H}$ NMR $\left(\mathrm{CDCl}_{3}, 400 \mathrm{MHz}\right) \delta$ 7.53-7.49 (m, 2H), 7.26-7.22 (m, 2H), $4.83(\mathrm{br} \mathrm{s}, 1 \mathrm{H}), 4.71$ (quint, $1 \mathrm{H}, J=6.8 \mathrm{~Hz}), 4.50(\mathrm{~d}, 1 \mathrm{H}, J=10.8 \mathrm{~Hz}), 4.49(\mathrm{~d}, 1 \mathrm{H}, J=10.8 \mathrm{~Hz}), 1.61(\mathrm{~d}, 3 \mathrm{H}, J=$ $6.8 \mathrm{~Hz}) \mathrm{ppm} ;{ }^{13} \mathrm{C} \mathrm{NMR}\left(\mathrm{CDCl}_{3}, 100 \mathrm{MHz}\right) \delta 140.4,132.0,128.0,122.1,93.2,78.0,54.3,22.7 \mathrm{ppm}$; IR (thin film) $v$ $3289,3046,1489,1433,1363,1181,1123,1077,1012,977,854,759 \mathrm{~cm}^{-1}$; HRMS $\left(E^{+}\right)$calcd for $\mathrm{C}_{10} \mathrm{H}_{11} \mathrm{BrCl}_{3} \mathrm{NO}_{3} \mathrm{~S} 408.8709$ found $431.8605\left(\mathrm{MNa}^{+}\right)$.

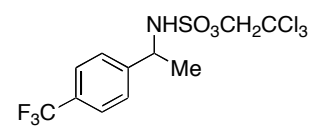

Purified by chromatography on silica gel (gradient elution: $11: 1 \rightarrow 4: 1$ hexanes/EtOAc); clear oil (40\%): TLC $_{f}=$ 0.40 (4:1 hexanes/EtOAc); ${ }^{1} \mathrm{H}$ NMR $\left(\mathrm{CDCl}_{3}, 400 \mathrm{MHz}\right) \delta 7.65$ (d, 2H, $\left.J=8.2 \mathrm{~Hz}\right), 7.49$ (d, 2H, $\left.J=8.2 \mathrm{~Hz}\right), 4.95$ (br d, $1 \mathrm{H}, J=7.0 \mathrm{~Hz}), 4.81$ (quint, $1 \mathrm{H}, J=7.0 \mathrm{~Hz}), 4.51(\mathrm{~d}, 1 \mathrm{H}, J=10.8 \mathrm{~Hz}), 4.49(\mathrm{~d}, 1 \mathrm{H}, J=10.8 \mathrm{~Hz}), 1.64(\mathrm{~d}, 3 \mathrm{H}, J=$ $7.0 \mathrm{~Hz}$ ) ppm; IR (thin film) v 3287, 2925, 1622, 1435, 1365, 1327, 1181, 1127, 1103, 1070, 1016, 977, 881, 843, $762 \mathrm{~cm}^{-1}$; HRMS $\left(\mathrm{ES}^{+}\right)$calcd for $\mathrm{C}_{11} \mathrm{H}_{11} \mathrm{Cl}_{3} \mathrm{~F}_{3} \mathrm{NO}_{3} \mathrm{~S} 398.9477$ found $421.9387\left(\mathrm{MNa}^{+}\right)$.

\section{$\mathrm{Ar}=p-\mathrm{RC}_{6} \mathrm{H}_{4} \mathrm{Et}$}

$\begin{array}{lrrc}\underline{\mathbf{R}} & \underline{\mathbf{\sigma}^{+}} & \underline{\underline{\boldsymbol{\sigma}}_{\mathbf{P}}} & \text { product ratio } \mathbf{A r} / \mathbf{C}_{\underline{0}} \underline{\underline{\mathbf{H}}} \underline{\underline{\mathbf{E}}} \underline{\mathbf{E}} \\ \mathrm{MeO} & -0.78 & -0.27 & 5.0 / 1.0 \\ { }^{\mathrm{t}} \mathrm{Bu} & -0.26 & -0.20 & 2.3 / 1.0 \\ \mathrm{Br} & 0.15 & 0.23 & 1.0 / 1.0 \\ \mathrm{CF}_{3} & 0.61 & 0.54 & 1.0 / 2.3 \\ \mathrm{NO}_{2} & 0.79 & 0.78 & 1.0 / 2.6\end{array}$

\section{Hammet plot fit against $\sigma^{+}$values}

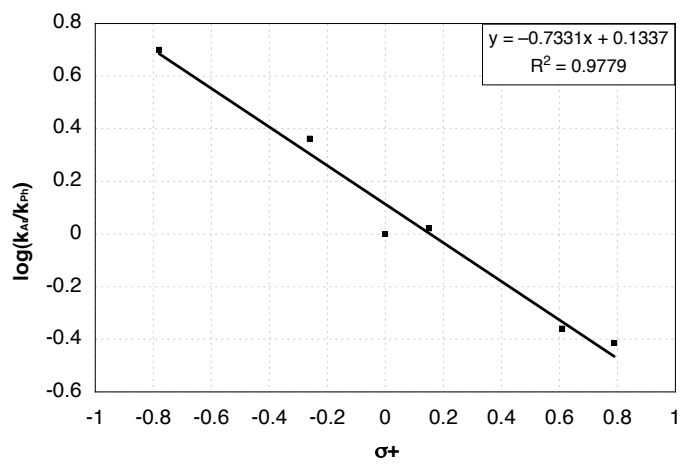

\section{Hammet plot fit against $\sigma_{\mathrm{p}}$ values}

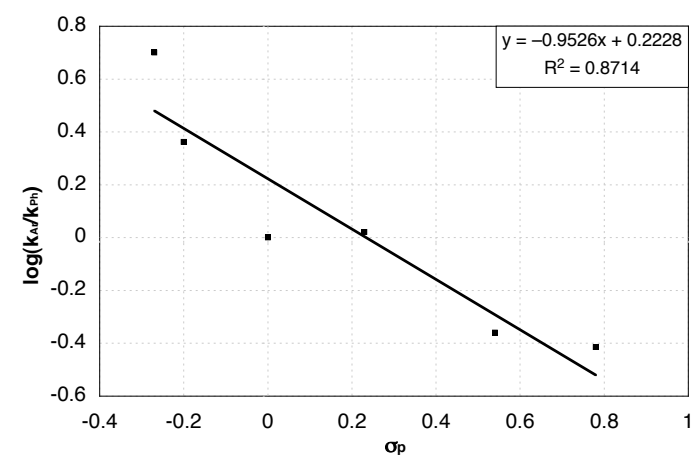




\section{UV-visible spectroscopic data}

$0.50 \mathrm{mM} \mathrm{Rh}_{2}(\mathrm{esp})_{2}$ in $\mathrm{CH}_{2} \mathrm{Cl}_{2}$

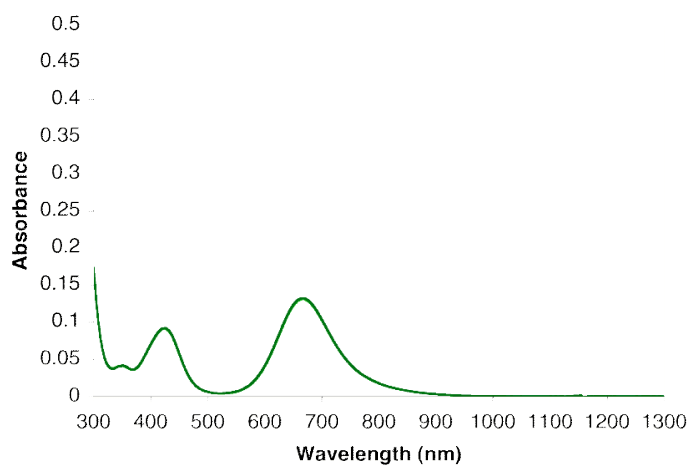

$0.50 \mathrm{mM} \mathrm{Rh}{ }_{2}(\mathrm{esp})_{2}+\mathrm{TcesNH}_{2}+\mathrm{PhI}\left(\mathrm{O}_{2} \mathrm{C}^{\mathrm{t}} \mathrm{Bu}\right)_{2}$ in $\mathrm{CH}_{2} \mathrm{Cl}_{2}$ after $2 \mathrm{~h}$

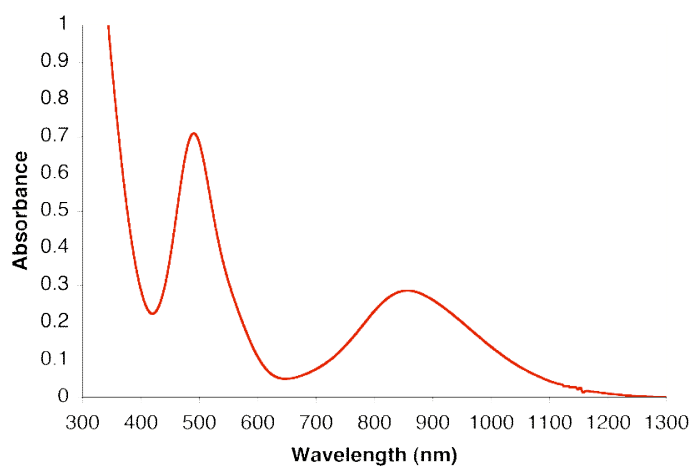

(1) Espino, C. G.; Fiori, K. W.; Kim, M.; Du Bois, J. J. Am. Chem. Soc. 2004, 126, 15378-15379.

(2) (a) Stang, P. J.; Boehshar, M; Wingert, H.; Kitamura, T. J. Am. Chem. Soc. 1988, 110, 3272-3278. (b) Merkushev, A. S.; Novikov, A. N.; Makachenko, S. S.; Moskal'chuk, A. S.; Gluskova, V. V.; Kogai, T. I.; Polyakova, L. G.; J. Org. Chem USSR (Engl. Transl.) 1975, 1246-1249.

(3) Guthikonda, K.; Du Bois, J. J. Am. Chem. Soc. 2002, 124, 13672-13673.

(4) $\mathrm{Cl}_{3} \mathrm{CCH}_{2} \mathrm{OSO}_{2} \mathrm{Cl}$ was prepared following a known procedure, see: (a) Hedayatullah, M.; Leveque, J. C.; Denivelle, L. C. R. Acad. Sc. Paris, C 1972, 274, 1937-1940. (b) Liu, Y.; Lien, I. F.; Ruttgaizer, S.; Dove, P.; Taylor, S. D. Org. Lett. 2004, 4, 209-212.

(5) Thiourea is used to decomplex any remaining $\mathrm{Rh}_{2}(\operatorname{esp})_{2}$ catalyst, thereby facilitating product purification. 